\section{Napoleon's hat}

SIR - Podsiadlowski et al. ' propound an interesting double-cone model of the circumstellar nebula to explain the "Napoleon's hat" on the north side and other light echoes observed around supernova 1987A. Although they offer various predictions, they do not mention an important datum which was obtained some time ago: the spectroscopic radialvelocity gradient in the small central ring ${ }^{2-6}$. Their model cannot accommodate this datum.

The ring ${ }^{6}$, observed with the Hubble Space Telescope (HST) and from the ground, appears elliptical on the plane of the sky, with its major axis (1.7 arcsec) roughly east-west. Presumably it is a circular ring, inclined by about $43^{\circ}$ so that either the north or the south point is closest to Earth. The radial-velocity gradient is small from east to west, but is positive from north to south. The spectroscopic data and images taken in best seeing $(<1 \operatorname{arcsec})^{3.5}$ show that near the slit the surface brightness of the outer loops is small compared to that of the ring itself. Emission by the outer loops is indeed seen in the spectra but is weak. Therefore the observed velocity gradient is likely to be dominated by the velocity field of the ring itself. Supporting this is the fact that the observed gradient is coterminous with the ring's direct image $^{5}$. The data are fitted well ${ }^{3-5}$ by a circular ring expanding radially from the progenitor star (or contracting onto it). With the reasonable assumption that the ring is expanding rather than contracting, the observers conclude that the north point is closest to $\mathrm{Earth}^{3-5}$.

Then the ring's plane is orthogonal to that shown for it in Fig. $2 a$ of ref. 1. This is a serious discrepancy. In the geometrical and dynamical model of Podsiadlowski et al., the ring was an equatorial belt or waist with symmetry axis roughly coinciding with that of the double cone. It was the remnant of an excretion disk produced in the orbital plane of the binary progenitor - a binary which also created the double cone. The doublecone geometry to explain Napoleon's hat, it seems, can be salvaged only by assuming either that the central ring somehow exists independently at the centre of the double-cone structure, with an axis orthogonal to the cone axis, or that the ring is now contracting onto the position of the binary progenitor which created it, rather than expanding. Neither assumption seems consistent with their dynamical model.

In a somewhat similar model by Wang and Mazzali $^{7}$, the predicted surface brightness on the plane of the sky is symmetrical north-south (in their approximation), so the axis of the model can be flipped to match the ring's axis.
The same is true of a paper by Luo and $\mathrm{McCray}{ }^{8}$, in which the ring's orientation is correct though mis-stated in the first paragraph. Neither paper ${ }^{7.8}$ contains an explanation of Napoleon's hat, and none of these models ${ }^{1,7,8}$ can do so when the ring is oriented correctly. As Kapteyn pointed out long ago, light echoes such as these contain much information about the circumstellar medium.

\section{JAMES E. FELTEN}

Code 685

ELI DWEK

NASA Goddard Space Flight Center,

Greenbelt, Maryland 20771, USA

PODSIADLOWSKI ET AL. REPLY - Felten and Dwek raise an important point, but they oversimplify the situation and we do not agree with their conclusions concerning the Napoleon's hat nebula. Although the velocity gradient is observationally well established, its implication for the inferred orientation of the ring is model-dependent. The problem is that the spatial resolution of the spectroscopic observations is usually larger (in most cases much larger) than the north-south extent of the ring $(\sim 1 ")$. Therefore, an overall velocity gradient would determine the orientation of the ring unambiguously only if there were no other significant contributions to the observed lines from elsewhere.

In contrast to Felten and Dwek, Wampler et al. ${ }^{9}$ find that the two outer loops surrounding the inner ring contribute significantly to the observed line fluxes (in O[III] the flux in the loops is one half the flux of the ring). Because of poor spatial resolution, the measured velocity gradient in a particular line can easily be dominated by the loops, whenever the flux in the loops is larger than about $10-20 \%$ and the characteristic radial velocity in the loops is larger than the radial velocity in the ring (the latter is model-dependent, but easily fulfilled in our model).

Therefore, an overall velocity gradient alone contains very little information about the orientation of the ring. The real information on the orientation can, however, be found in the detailed spectral line shapes (see, for example, ref. 5). Indeed, these may suggest an orientation of the ring different from the one we assumed. But a realistic model has to include the emission from the loops and the possible clumpiness of material in the ring as well as in the loops and should be able to reconcile some of the apparent discrepancies in the reported observations noted above.

A second even more important point is that, even if the ring's orientation were orthogonal to the orientation used in our figures, this would not at all affect our geometrical model for the Napoleon's hat nebula, which was the main result of our paper (our geometrical model does not require any particular orientation of the ring). In particular, we would like to re-emphasize that our model has several unique features. In particular, we showed that the rim of Napoleon's hat has to have the same topology as the intersection of an elliptical cone with a paraboloid (this is entirely model-independent). Of course, Felten and Dwek are correct that a different orientation of the ring would make our suggested dynamical model for the whole nebula less attractive. Although it would not necessarily invalidate it, a different, probably more complicated, dynamical model would be required.

A real test of our model therefore has to come either from high-resolution images with the New Technology Telescope (NTT) or the HST or from detailed light echo studies (for example, the evolution of the inner light echo ${ }^{10}$ should directly map out the predicted cone structure). A recent picture ${ }^{11}$ taken with the NTT shows that our emission model was not realistic. But because the particular emission model was chosen only for illustrative purposes, this is not important. What is important is the location of the northern end of the Napoleon's hat structure and the appearance of a southern cone (provided that it is not obscured). Another critical feature is the extension of the inner loops within the central ring. Close inspection of the HST image ${ }^{12}$ shows and the earlier NTT image $^{9}$ hints that the northern loop is closed, while the southern loop is not (both of which are consistent with our model). By now, our model predicts that the southern loop should also appear closed. A potential problem with this is that the HST may not have the dynamic range and the NTT the necessary resolution to resolve this question unambiguously.

\section{PHILIPP PODSIADLOWSK ANDREW C. FABIAN IAN R. STEVENS}

Institute of Astronomy,

University of Cambridge,

Madingley Rd,

Cambridge $\mathrm{CB} 3 \mathrm{OHA}$, UK.

1. Podsiadiowski, P., Fabian, A. C. \& Stevens, I. R. Nature 354, 43-46 (1991)

2. Wood, P. R. \& Faulkner, D. J. IAU Circ. No. 4739 (1989)

3. Crotts, A. P. S. in Supernova 1987A and Other Supernovae (eds Danziger, I. J. \& Kjär, K.) 559-567 (ESO Garching, 1991).

4. Meikle W. P. S et al, in Supernova 1987A and Other Supernovae (eds Danzinger, I. J. \& Kiär, K.) 595-603 (Eso, Garching, 1991)

5. Crotts, A. P. S. \& Heathcote, S. R. Nature $\mathbf{3 5 0}$ 683-685 (1991)

6. Dwek, E. \& Felten, J. E. Astrophys. J. 387, 551 (1992)

7. Wang, L. \& Mazzali, P. A. Nature 355, 58-61 (1992)

8. Luo, D. \& McCray, R. Astrophys. J. 379, 659 (1991)

9. Wampler, E. J. et al. Astrophys. J. 362, L13 (1990).

10. Crotts, A. P. S. \& Kunkel, W. E. Astrophys J. 366, L73-L77 (1991).

11. Gouiffes, C. \& Della Valle, M. The Messenger, ESO 66 , 34-35 (1991)

12. Jacobson, P. et al. Astrophys. J. 369, L63-L66 (1991) 